\title{
Seroprevalence of IgG antibodies against measles in a selected Polish population - do we need to be re-vaccinated?
}

\author{
JUSTYNA HOLKA ${ }^{1}$, KATARZYNA PAWLAK ${ }^{2}$, OLGA CIEPIELA ${ }^{2,3}$ \\ ${ }^{1}$ Students Scientific Group of Laboratory Medicine, Medical University of Warsaw, Warsaw, Poland \\ ${ }^{2}$ Central Laboratory, Central Teaching Hospital of University Clinical Center, Warsaw, Poland \\ ${ }^{3}$ Department of Laboratory Diagnostics, Medical University of Warsaw, Warsaw, Poland
}

\begin{abstract}
Introduction: Measles is highly contagious, but preventable viral disease. Its outbreaks appear all over the world, and decreasing herd immunity excludes its elimination. High levels of IgG antibodies against a virus efficiently protects against infection.

Aim of this study: To assess the seroprevalence of anti-measles IgG in the serum of patients at different age levels tested for measles IgG at our laboratory.

Material and methods: The study was conducted from March to June of 2019. Retrospective analysis included results for measles-specific IgG from 364 tested patients. The age of enrolled subjects ranged from four months to 101 years, with a median age of 46 , and a mean age of $43 \pm 18$. Quantification of anti-measles $\operatorname{Ig} G$ was performed using indirect chemiluminescence immunoassays on the

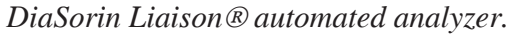

Results: Our results showed a seropositivity ratio of $78.02 \%$. The lowest number of seropositive subjects was in the group of infants (0-1 years old), with a ratio of $53.85 \%$, and the group of adults of 19-38 years old at 55.68\%. The group of the oldest patients (70-101 years old) had the highest ratio of seropositive subjects (100\%), while adults of 60-69 years old had a seropositivity ratio of $97.22 \%$.

Conclusions: These data suggest that the group of young adults who were vaccinated with one or two doses of MMR vaccine in childhood are the most susceptible for infection, and when working in contact with other people, should be re-vaccinated for protection against measles.
\end{abstract}

Key words: anti-measles IgG, herd immunity, humoral immunity, measles, seroepidemiology.

(Centr Eur J Immunol 2019; 44 (4): 380-383)

\section{Introduction}

Measles is a highly contagious viral disease that causes a high-rate of morbidity and mortality. Effective vaccination with measles-mumps-rubella (MMR) vaccine made this disease easily preventable. Despite widespread accessibility to the vaccine and obligatory vaccination with MMR at the age of 12-13 months and again at 6-10 years of age (depending on the country), anti-vaccine movements have contributed to a decrease in vaccine coverage. Efficient herd immunity can be obtained when the vaccine coverage exceeds 95\% [1-3]. Data from the National Institute of Public Health - National Institute of Hygiene (NIPH-NIH) indicate an increasing ratio of diagnosed measles; the number of measles cases in 2018 was 5-times higher than that in 2017 (339 vs. 63) [4]. Measles outbreaks are now occurring all around the world as a response to decreased herd immunity. According to the European Centre for Disease Prevention and Control (ECDC), vaccination coverage is below 95\% in most European countries, and the highest number of confirmed cases were reported in France, Italy, Bulgaria, Lithuania, and Poland, with more than 100 cases in April of 2019 [5].

In 2010, the World Health Organization, Office for $\mathrm{Eu}-$ rope, undertook a resolution signed by all countries in the WHO European region, to eliminate measles and achieve sufficient herd immunity [6]. Unfortunately, in Poland the ratio of vaccination, which should have increased, continues to decrease with a ratio below $93 \%$ in 2018 [4].

Measles virus infection causes life-long protective immunity, while two doses of MMR vaccine should also provide appropriate immunization [3]. However, there are some reports suggesting that the immune response to measles following MMR vaccination provides less-sufficient

Correspondence: Olga Ciepiela, PhD, Department of Laboratory Diagnostics, Medical University of Warsaw, 1A Banacha St., 02-097 Warsaw, Poland, e-mail: olga.ciepiela@wum.edu.pl Submitted: 6.08.2019; Accepted: 9.09.2019 
immunization and that the amount of specific anti-measles immune antibodies (IgG class) decreases over time [7, 8].

The aim of this study was to analyze the seroprevalence of anti-measles IgG antibodies among patients referred for testing in a central laboratory of the Central Teaching Hospital of University Clinical Centre in Warsaw, Poland.

\section{Material and methods}

\section{Analyzed data}

The study was conducted from March to June of 2019. Retrospective analysis included results for measles-specific IgG from 364 tested patients. The age of enrolled subjects ranged from four months to 101 years, with a median age of 46 and a mean age (SD) of $43 \pm 18$. There were a total of 245 women and 118 men tested. Adult patients, who were tested with regard to measles-specific IgG, were either health care workers or patients who were suspected of having contact with active measles virus within the last 48 hours. All analyzed data were anonymized; the basis of study was age, sex of patient, and the reactivity of anti-measles IgG measured using commercial Liaison ${ }^{\circledR}$ indirect chemiluminescence immunoassay on the DiaSorin Liaison ${ }^{\circledR}$ automated analyzer, which has been proven highly specific and sensitive in the detection of measles-specific IgG [9]. The threshold value regarded as protective immune status was $16.5 \mathrm{AU} / \mathrm{ml}$ [border line 13.516.5 AU/ml; lower limit of detection (LoD) at $5 \mathrm{AU} / \mathrm{ml}$ and higher LoD at $300 \mathrm{AU} / \mathrm{ml}$. Study was accordant with the regulation of Bioethics Committee of Medical University of Warsaw.

\section{Statistical analysis}

Data analysis was performed using GraphPad Prism v.6 (GraphPad Software, La Jolla, CA, USA). The type of data distribution was analyzed using the D'Agostino-Pearson omnibus normality test. The seroprevalence was analyzed using the modified Wald method. Differences between studied groups were evaluated using the
Kruskal-Wallis test. The Mann-Whitney test was used to compare the serological status between men and women. The $\chi^{2}$ or Fischer exact tests were used to compare the seropositivity rates between different groups. A $p$-value less than 0.05 was considered statistically significant.

\section{Results}

Total number of 284 patients had measles-specific IgG antibodies concentrations higher than $16.5 \mathrm{AU} / \mathrm{ml}$, thus the seropositivity was $78.02 \%$ (95\% CI: $73.48-81.98$ ). The study group was divided into seven age groups, based on their possible MMR vaccination: 1$) 0-1$ years old $(n=13$, before obligatory vaccination at age 13-14 month); 2) 1.3-16 years old ( $n=17$, children who should be vaccinated with two doses of MMR vaccine); 3) 19-38 years old ( $n=88$, young adults who should be vaccinated with two doses of MMR vaccine); 4) 39-45 years old ( $n=61$, adults who should be vaccinated with one dose of MMR vaccine); 5) 46-59 years old ( $n=131$, adults who were not covered by an obligatory vaccination programme, occupationally active); 6) 60-69 years old ( $n=36$, adults who were not covered by an obligatory vaccination programme, partially occupationally active); 7) 70-101 years old ( $n=16$, adults who were not covered by an obligatory vaccination programme, retired). In the group of adult subjects, the percentage of seropositivity was estimated at $80.12 \%$ (95\% CI: 75.48-84.07). The distribution of anti-measles IgG in each age group is presented in Table 1.

There was a significant difference in anti-measles IgG concentration between men and women, indicating that men have a lower level of specific anti-measles antibodies (median values 145.5 vs. $300 \mathrm{AU} / \mathrm{ml}, p=0.033$ ). An age dependence of antibody concentrations was observed (Fig. 1) and divided into three groups based on distribution: two with the lowest and one with the highest concentration of antibodies. The first group included patients with the lowest values in the $0-1$ years old group. The second group included 20-45 years old patients, and the third group included patients older than 45 years, in which anti-measles $\mathrm{IgG}$ concentrations reached the highest values

Table 1. Characteristic of studied groups with exact results of measles-specific IgG prevalence and values

\begin{tabular}{lcccccc}
\hline $\begin{array}{l}\text { Age group } \\
(\text { years old })\end{array}$ & $\begin{array}{c}\text { Male } \\
(\boldsymbol{n})\end{array}$ & $\begin{array}{c}\text { Female } \\
(\boldsymbol{n})\end{array}$ & $\begin{array}{c}\text { IgG positive } \\
(\boldsymbol{n})\end{array}$ & $\begin{array}{c}\text { IgG negative } \\
(\boldsymbol{n})\end{array}$ & $\begin{array}{c}\text { Median IgG concentration } \\
(\mathbf{A U} / \mathbf{m l})\end{array}$ & $\begin{array}{c}\text { Seroprevalence } \\
(\boldsymbol{\%} \text { [95\% } \mathbf{C I}])\end{array}$ \\
\hline $0-1$ & 13 & 0 & 7 & 6 & 19.8 & $53.85(29.13-76.81)$ \\
\hline $1.3-16$ & 8 & 9 & 11 & 6 & 28.6 & $64.71(41.16-82.83)$ \\
\hline $19-38$ & 38 & 50 & 49 & 39 & 39.05 & $55.68(45.28-65.61)$ \\
\hline $39-45$ & 15 & 46 & 44 & 17 & 103 & $72.13(59.76-81.88)$ \\
\hline $46-59$ & 30 & 101 & 122 & 9 & 300 & $93.13(87.30-96.51)$ \\
\hline $60-69$ & 7 & 29 & 35 & 1 & 300 & $97.22(84.58->99.99)$ \\
\hline $70-101 *$ & 8 & 8 & 16 & 0 & 300 & $100(77.31-100.00)$ \\
\hline
\end{tabular}




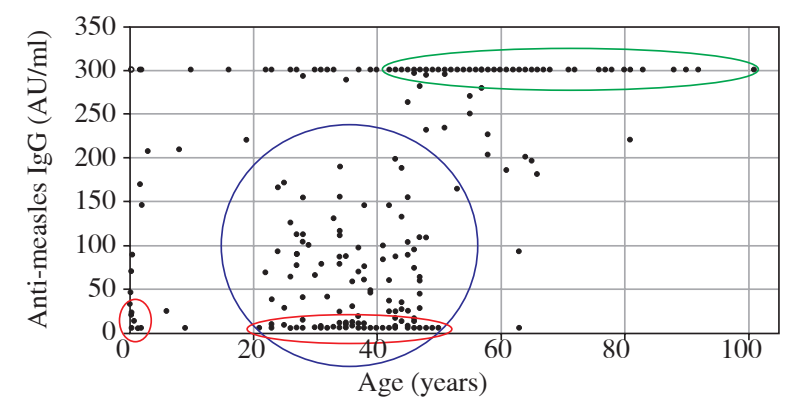

Fig. 1. Distribution of IgG anti-measles antibodies according to patient's age. Red circles show the population with seronegativity, the green circle shows the group with the highest level of immunization, and the blue group showed the highest diversity

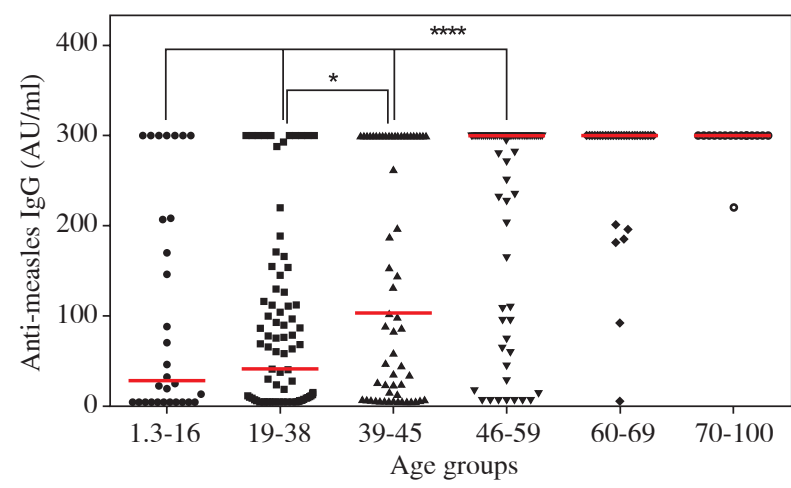

Fig. 2. Median values of measles-specific IgG concentration is selected patient groups $\left({ }^{*} p<0.05,{ }^{* * * *} p<0.0001\right)$

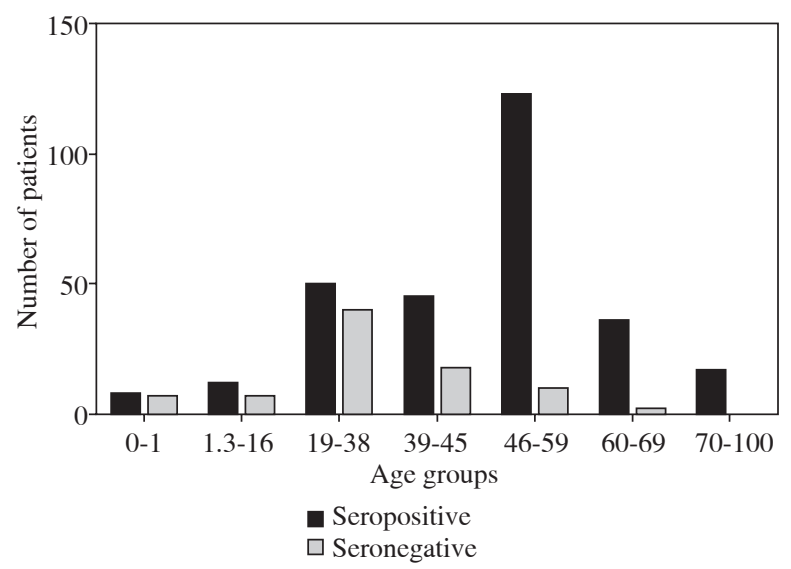

Fig. 3. Numbers of seropositive and seronegative subjects in the studied age groups. The difference is statistically significant, $p<0.0001$

(Fig. 1). Individuals aged 20-45 had the most diversified distribution of results.

A significantly higher number of seronegative patients were in the male group than in the women group
( $p=0.04)$. The studied groups differed significantly in measles-specific IgG values, which are presented in Table 1 and Figure 2. There was also a significant difference in the seronegativity/seropositivity ratio between examined aged groups, with the lowest seropositivity ratio in the group of subjects that were 19-38 years old (Fig. 3).

\section{Discussion}

The results showed that $78.02 \%$ of patients enrolled in the study had positive titres of measles-specific IgG antibodies. The lowest ratio of seropositivity was found in a group of adult patients who were covered by an immunization schedule with two doses of MMR vaccination in childhood, and in a group of children aged 0-1 who were not covered by vaccination. This observation is consistent with the results from other studies [10]. According to the immunization schedule appropriate for each study group, a second vaccination should have been administered at the age of 7-10, which means that the time between the last vaccination and the test for the presence of specific IgG was 9-28 years. Interestingly, the seropositivity ratio was higher in a group that, according to immunization schedule, should have administered one dose of MMR; however, we cannot exclude that this group also involved non-vaccinated subjects, since coverage of vaccination in 1978 was estimated at 50\% and in 1981 it was $~ 80 \%$ [11]. The seropositivity ratios observed in our study between vaccinated once and twice are not accordant with the results from a study performed in Japan [12]. Here, we show a significantly lower titer of antibodies in the group vaccinated twice than after one dose of MMR. In addition, the seropositivity ratio was the highest in all groups, which were not vaccinated due to natural immunization caused by the disease. Nevertheless, the number of seropositive subjects was quite low compared with that of other European studies $[2,3,13]$, which does not confirm that immunogenicity from the measles vaccine has life-long effects [14]. We observed that a group of patients who were covered by immunization schedule had significantly lower titres of antibodies against measles that a non-vaccinated older group, which is consistent with the results from previous studies $[3,8,13,15]$. Also, our results showed a similar distribution of anti-measles IgG among different age groups as shown by Ristic et al. [13]. Here, we observed the same trend of decreasing measles-specific IgG titer over time from vaccination, as reported by Kennedy et al. [7]; however, there was a longer time between possible vaccination and serological test in our study (up to 40 vs. 17 years). Our results are consistent with those obtained by Coppeta et al. who showed that total seropositivity reached $87 \%$ in a group of adult hospital employees, with the highest ratio in a group of patients not vaccinated in childhood (born before 1982) and lowest in the group born in 1992-1999 [1]. In contrast to previous studies, our results showed 
a statistically significant difference in seropositivity ratios between men and women [1-3, 10, 16, 17]. Higher immunization status in women might be associated with additional vaccination against rubella, which was administered together with vaccine against mumps and measles later in childhood. We observed an alarming susceptibility to disease in 56/149 (37.58\%) individuals who were $19-45$ years old, and this rate is almost twice that reported in other studies $[3,13,18]$ and similar to the results reported in studies conducted in South Korea, Italy, and Czech Republic [14, 15, 19]. These data strongly suggest that this age group is at particular risk of possible infection and should be re-vaccinated. This includes health care workers, teachers, or others who work with people who might be a reservoir of the virus. However, as it was suggested by the Italian National Plan for Measles and Congenital Rubella Elimination, foreign-born women who are registered for the first time in the Italian healthcare system, should be additionally vaccinated [18].

\section{Conclusions}

The main limitation of this study is a lack of information about vaccination status among enrolled subjects. Given the retrospective nature of this study and patients' personal data anonymized, we were not able to obtain such medical records. Despite this limitation, our results are reliable and consistent with those of other national [11] and European studies. These results warrant further discussion about modification of immunization schedules with regard to recommended vaccines in adulthood. Such a suggestion concerning vaccination against mumps, which is administered with the MMR vaccine, has already been raised [20]. From an economical point of view, it should be considered if preventive vaccination is more cost-effective than previous screening of specific anti-measles IgG antibodies in the most susceptible groups. Studies performed on mumps immunization strategy in Italy showed that screening for antibodies and re-vaccination of individuals who were seronegative only is less expensive than vaccination without confirmation of serological status [21]. An appropriate analysis should be performed with regard to measles immunization, given that the seropositivity ratio in young adults is not sufficient to retain expected herd immunity level.

The authors declare no conflict of interest.

\section{References}

1. Coppeta L, Pietroiusti A, Lieto P, et al. (2019): Measles immunity in an Italian teaching hospital. Occup Med (Lond) 69: 143-145.

2. Gallone MS, Germinario C, Larocca A, Tafuri S (2017): Long time immunogenicity of measles vaccine in the vaccination era: An open question. Hum Vaccin Immunother 13: 117-119.
3. Lengyel G, Marossy A, Anosi N, et al. (2018): Screening of more than 2000 Hungarian healthcare workers' anti-measles antibody level: results and possible population-level consequences. Epidemiol Infect: 1-5.

4. Szczepienia info 2019. Available from: www.szczepienia. pzh.gov.pl.

5. Measles. Available from: https://ecdc.europa.eu.

6. World Health Organization Regional Office for Europe. WHO Regional Committee for Europe resolution EUR/RC60/ $\mathrm{R} 12$ on renewed commitment to elimination of measles and rubella. Copenhagen 2010.

7. Kennedy RB, Ovsyannikova IG, Thomas A, et al. (2019): Differential durability of immune responses to measles and mumps following MMR vaccination. Vaccine 37: 1775-1784.

8. Gidding HF, Quinn HE, Hueston L, et al. (2018): Declining measles antibodies in the era of elimination: Australia's experience. Vaccine 36: 507-513.

9. de Ory F, Minguito T, Balfagon P, Sanz JC (2015): Comparison of chemiluminescent immunoassay and ELISA for measles IgG and IgM. APMIS 123: 648-651.

10. Tomaskova H, Zelena H, Kloudova A, Tomasek I (2018): Serological survey of measles immunity in the Czech Republic, 2013. Cent Eur J Public Health 26: 22-27.

11. Janaszek-Seydlitz W. Odra w Polsce - badania seroepidemiologiczne. Państwowy Zakład Higieny, Warszawa 2004.

12. Shoho Y, Kimura T, Yanagawa Y, et al. (2018): Vaccination Status and Antibody Titers against Rubella and Measles among Japanese Female College Students Majoring in Childcare between 2015 and 2018. Tohoku J Exp Med 246: 73-79.

13. Ristic M, Milosevic V, Medic S, et al. (2019): Sero-epidemiological study in prediction of the risk groups for measles outbreaks in Vojvodina, Serbia. PLoS One 14: e0216219.

14. Smetana J, Chlibek R, Hanovcova I, et al. (2017): Decreasing Seroprevalence of Measles Antibodies after Vaccination - Possible Gap in Measles Protection in Adults in the Czech Republic. PLoS One 12: e0170257.

15. Ledda C, Cina D, Garozzo SF, et al. (2019): Vaccine-preventable disease in healthcare workers in Sicily (Italy): seroprevalence against measles. Future Microbiol 14: 33-36.

16. Zhang Z, Chen M, Wang Y, et al. (2019): Seroepidemiology of measles in Beijing, China: a cross-sectional study. Hum Vaccin Immunother 15; 2112-2116.

17. Jablonka A, Happle C, Wetzke M, et al. (2017): Measles, Rubella and Varicella IgG Seroprevalence in a Large Refugee Cohort in Germany in 2015: A Cross-Sectional Study. Infect Dis Ther 6: 487-496.

18. Tafuri S, Gallone MS, Gallone MF, et al. (2016): Monitoring the process of measles elimination by serosurveillance data: The Apulian 2012 study. Vaccine 34: 2092-2095.

19. Chang HH, Kim SW, Kwon KT, et al. (2019): Preliminary Report of Seroprevalence of Anti-Measles Immunoglobulin G among Healthcare Workers of 6 Teaching Hospitals of Daegu, Korea in 2019. Infect Chemother 51: 54-57.

20. Kaaijk P, Wijmenga-Monsuur AJ, van Houten MA, et al. (2019): A Third Dose of Measles-Mumps-Rubella Vaccine to Improve Immunity Against Mumps in Young Adults. J Infect Dis 2019; pii.jiz.188. doi: 10.1093/infdis/jiz188

21. Coppeta L, Balbi O, Baldi S, et al. (2019): Pre-vaccination IgG screening for mumps is the most cost-effectiveness immunization strategy among health care workers. Hum Vaccin Immunother 15: 1135-1138. 